\title{
Fotonic and Electron Microscopy Images for Quality Evaluation of Delignification of Agave Fibers
}

\author{
Hilda Hernández-Hernández ${ }^{1}$, Jorge Chanona-Pérez ${ }^{1, *}$, \\ Georgina Calderón-Domínguez ${ }^{1}$, Eduardo Terrés Rojas ${ }^{2}$, M.J. Perea-Flores ${ }^{3}$, \\ Jorge Mendoza-Pérez ${ }^{4}$, Alberto Vega ${ }^{5}$, and Pablo Ligero ${ }^{5}$
}

${ }^{1}$ Departamento de Ingeniería Bioquímica, Escuela Nacional de Ciencias Biológicas, Instituto Politécnico Nacional, Plan de Ayala y Carpio s/n, Col. Santo Tomas, C.P. 11340, México D.F.

\{hilda2hdez, jorge_chanona, ginacaldero\}@hotmail.com

${ }^{2}$ Laboratorio de Microscopía Electrónica de Ultra Alta Resolución. Instituto Mexicano del Petróleo (IMP). Eje Central Lázaro Cárdenas N.152, Edif. 33. Colonia San Bartolo Atepehuacan. C.P. 07730. México D.F. eterres@imp.mx

${ }^{3}$ Centro de Nanociencias y Micro-Nanotecnología. Instituto Politécnico Nacional. Luis Enrique Erro s/n, Unidad Profesional Adolfo López Mateos, Col. Zacatenco, C. P. 07738, México D.F. peflo_ma@hotmail.com

${ }^{4}$ Departamento Ingeniería en Sistemas Ambientales. Escuela Nacional de Ciencias Biológicas. Instituto Politécnico Nacional. Wilfrido Massieu s/n U. Profesor Adolfo López Mateos,

Gustavo A. Madero, 07738 México, D.F.

jorgemendozaperez@yahoo.com

${ }^{5}$ Departamento Química Física e Enxeñería Química I. Facultade de Ciencias. Universidade da Coruña. Campus da Zapateira. 15071 A Coruña España

\{devega, pligero\} @udc.es

\begin{abstract}
The present work is aimed to study the microstructural changes that occurred during the delignification process of agave fibers using microscopy techniques and image analysis (AI). Acetosolv kinetic was followed by chemical analysis and by light (LM), confocal laser scanning (CLSM), and scanning electron (SEM) microscopies, evaluating the micrographs by image analysis (IA). Kinetic studies showed that delignification process followed three stages: initial, bulk and residual; these stages kept a relation with the microstructural changes occurring in the fibers. The data obtained integrate numerical information that could be valuable for study of pulping of lignocellulosic materials and these techniques can be used as useful non-destructive methods for the evaluation of the delignification process.
\end{abstract}

Keywords: Agave atrovirens, microstructure fibers, pulping kinetics, Acetosolv.

* Corresponding author.

F. Huang and A. Sugimoto (Eds.): PSIVT 2013 Workshops, LNCS 8334, pp. 83-92, 2014.

(C) Springer-Verlag Berlin Heidelberg 2014 


\section{Introduction}

The Agave is a plant with about 166 species and Mexico is the most important center of biodiversity of these species. During the manufacturing processes of Agave beverages, the leaves are discarded in order to reach the plant's "core" or "piña" from which Agave plant is used to obtain fermentable sugars [1]. From this process, Agave residues could be used to obtain fiber for the production of paper, bioethanol and other chemicals. Organosolv processes, usually defined as the delignification methods where the reaction system is based on the use of organic acids and different catalyzers. These processes have proven to be promising in achieving a comprehensive utilization of lignocellulosic materials with minimal environmental impact. On the other hand, microscopy techniques have been useful to know the morphological characteristics of different lignocellulosic materials and Light (LM), confocal laser scanning (CSLM), scanning electron (SEM), transmission electron (TEM) and atomic force (AFM) microscopies have frequently been used to characterize the morphology, microstructure, topography, ultrastructure and microdistribution of lignocellulosic components in several plant tissues [2-4]. Nevertheless, these studies have been mainly focused to describe the morphology of lignocellulosic materials. On the other hand, images analysis of microscopy images could be a useful tool to have a quantitative analysis of several biological materials, including the lignocellulosic plants [5-7]. Such studies have not been performed to describe the microstructural changes that occur during the delignification process. Therefore, the aim of this work was to study the microstructural changes involved in the Acetosolv process of agave fibers by means of microscopy techniques and image analysis looking for a a better understanding of the process and their effect on the quality of the obtained fibres.

\section{Materials and Methods}

\subsection{Delignification Kinetic}

The delignification kinetic of Agave fibers were carried out by applying the Acetosolv process [8]. The kinetic of lignin release was followed by chemical analysis (Klason lignin, pulp yield and selectivity). The experiment was carried out in triplicates.

\subsection{Microscopy Study of Delignification Kinetic}

\section{Light Microscopy (LM)}

In order to evaluate the changes in the thickness of the fibers during the kinetic at least 10 fibers from each reaction time were observed with an optical microscope (10x, Nikon, Eclipse 50i, Japan). These micrographs were analyzed by images analysis (ImageJ v.1.42q, software National Institutes Health, Bethesda, MD, USA), converting the RGB images to binary by using the threshold tool in a range of 80-255. Fiber thickness was determined from 5 measurements from each image using the measurement tool of the software. 


\section{Confocal Laser Scanning Microscopy (CLSM)}

CLSM allows the simultaneous view of lignin and cellulose due to their native fluorescence; Two different laser emission sources producing different wavelengths were used for both materials (405 nm, lignin; $480 \mathrm{~nm}$ cellulose), In order to evaluate quantitatively the CLSM micrographs of the fiber delignification process, an image analysis methodology was implemented. Thereby, regions of interest (ROI's) of $80 x 80 \mu \mathrm{m}$ were taken from CLSM RGB images of cellulose and lignin. Subsequently, the ROI's were converted to grayscale. From these ROI's the average value of grey level (GL), using the ImageJ software histogram was extracted and also the image entropy was evaluated. In the present work the entropy of images was used as a measure of heterogeneity of the fluorescence and the GL value as a measure of the fluorescence intensity in the images. Additionally, gray scale images were converted to binary format by using threshold tool in a range between 20-255 units. From these images total fluorescence area was obtained and expressed as area fraction (AF) which represents a relative measure of cellulose content or lignin content in the ROI. At least 10 images for each reaction time of delignification process were used for extraction of imaging parameters. All steps of image analysis were performed in ImageJ.

\section{Environmental Scanning Electron Microscopy (ESEM)}

The fibers were mounted in an aluminum cylindrical sample holders provided with double sided carbon tape. Subsequently, they were coated with gold in an ionizer (Desk II, Denton Vacuum, Moorestown, NJ, USA) for $500 \mathrm{~s}$ at $100 \mathrm{mTorr}$ and 20 $\mathrm{mA}$. The samples were observed with an environmental scanning electron microscope (XL 30, Philips, USA) at 500x and $25 \mathrm{kV}$.

\subsection{Linear Prediction Models for Delignification and Statistical Analysis}

Imaging parameters values can be useful for proposing a non-destructive method to evaluate the delignification process. For this reason chemical and imaging parameters values obtained from pulping kinetics were analyzed by Pearson method to obtain linear prediction models. The relationships between kinetic study and image analysis results were selected based on the better linear prediction models. The criteria for selection of the better relationships was based on the highest correlation $(\mathrm{R})$ and determination $\left(\mathrm{R}^{2}\right)$ coefficients as obtained from Pearson and linear regression analysis (least squares approach) respectively. The statistical analysis was carried out using XLSTAT v2009.3.02 software (Addinsoft, 232 USA). Descriptive statistics and linear regressions were done for all experiments and for the image analysis results the normal distribution of the data (Shapiro-Wilk Kolmogorov-Smirnov) was performed to determine using the statistical program Sigmaplot version 12.0 (Systat software Inc., USA). 


\section{$3 \quad$ Results and Discussion}

\subsection{Microstructural Changes during Delignification Kinetics}

Figure 1 shows the delignification kinetic at $90 \%$ acetic acid and $1.5 \%$ solution $\mathrm{HCl}$ as catalyzer with the three delignification stages of agave fibers. The first period can be associated to a high reaction rate $(\mathrm{k})$ of $7 \times 10^{-4} \mathrm{~min}^{-1}$ that is explained by easy removal of superficial lignin in the agave fiber. This period is known as initial stage [12]. Regarding the second period or bulk stage a minor value of $\mathrm{k}\left(3 \times 10^{-4} \mathrm{~min}^{-1}\right)$ was obtained. This diminishing in the reaction rate corresponds to the removal of interfibrillar lignin of internal zones of the fibers. Finally, the residual stage showed the lowest value of $\mathrm{k}\left(1 \times 10^{-4} \mathrm{~min}^{-1}\right)$ due to that during this stage the lignin content in the microfibers is removed. These delignification stages were similar to those reported for the pulping of Eucalyptus globulus [12] and several hardwoods when using a Kraft process [13].

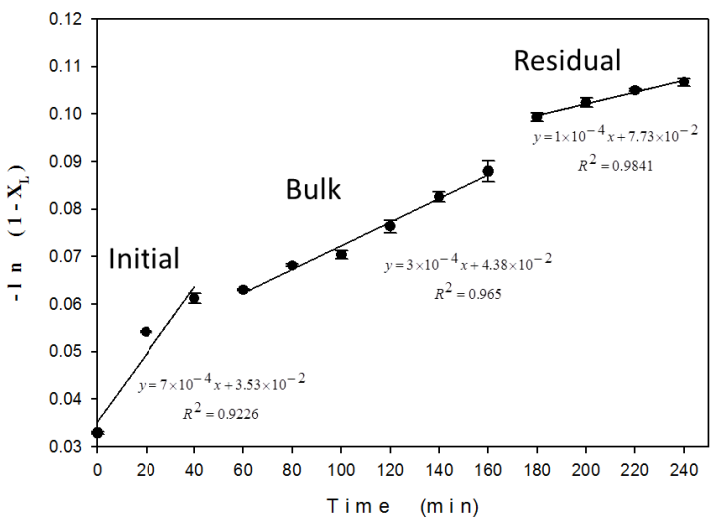

Fig. 1. Chemical reaction kinetic and delignification stages

For an additional description of the pulping process, the microstructural studies were related with the delignification kinetic process. Figure 2 shows the microstructural changes of fibers, observed under CLSM and SEM, for selected cooking times. Figures 2A-2C show CLSM images where two spectral channels can be simultaneously observed, and where the green color corresponds to lignin autofluorescence, while the blue color is linked with the fluorescence of calcofluor used for cellulose stained. At the begging of the kinetic $(0 \mathrm{~min})$ the fluorescence of the cellulose was the lowest (Figure 2A), while the lignin autofluorescence was very intense. This corresponds to lignified fibers with lower cellulose purification. Additionally, SEM image illustrates the structural arrangement of the fiber without damage (Figure 2D). After 120 minutes of reaction, the fibers showed an increase in the cellulose fluorescence and a decrease in the lignin autofluorescence, and where the distribution of these components in the fibers could be observed in similar proportions (Figure 2B). Structural damages on the cellular tissue, the collapse of the cell walls, as well as the 
presence of fractures, disruption of fibers clumps and a more porous structure, promoted by removal of interfibrillar or bulk lignin, were detected under SEM (Figure 2E). At 240 minutes the fibers showed a higher intensity of cellulose fluorescence which almost covered all the fibers surface. In contrast, the lignin autofluorescence intensity was lower (Figure 2C). Thereby, the lignin contained in the fibers was almost completely removed, indicating that the delignification process was adequate. SEM images also provided evidence of a major structural damage in the fibers, due to a deeper acetic acid infiltration through the fibers during the final delignification stage. In this step the lignin from microfibrils was eliminated generating porous and structural damages in the tracheids of fibers bunches (Figure 2F).

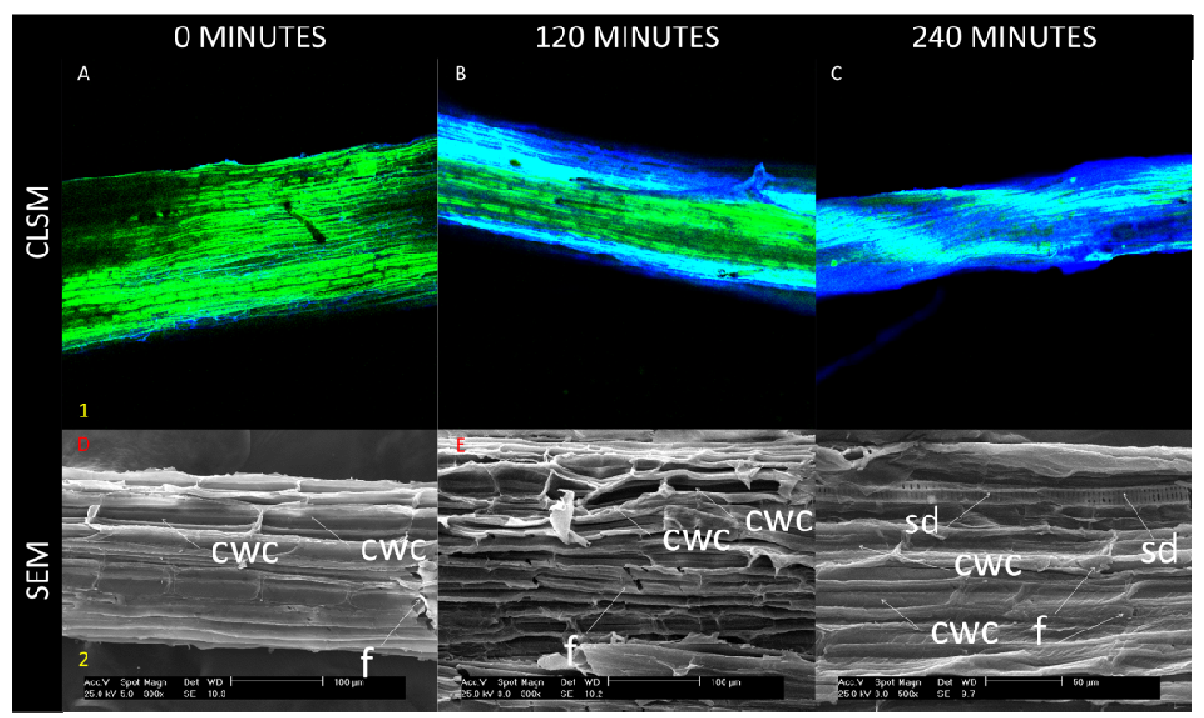

Fig. 2. CLSM images (A, B and C) and SEM images (D, E and F) of agave fibers at different times of pulping $(0,120$ and $240 \mathrm{~min})$. Green color corresponds to lignin and blue color to cellulose. Here cw: cell wall; f: fractures; cwc: cell walls collapse: sd: structural damage in tracheids.

Another important effect of the pulping process was the reduction of agave fibers thickness. As regard to the kinetic of agave fibers thickness reduction (Figure 3) it had a similar tendency to RL kinetic and also three stages were observed. The first stage yielded sizes of fibers between 592.3-505.6 $\mu \mathrm{m}$ with a rate of size reduction of 1.45 $\mu \mathrm{m} / \mathrm{min}$. In the second stage, the size of fibers varied from 481.3 to $410.4 \mu \mathrm{m}$ at a rate of $0.71 \mu \mathrm{m} / \mathrm{min}$, and finally at the third stage the fiber diameters varied from 275.6 to $309.1 \mu \mathrm{m}$ having a size reduction rate of $0.42 \mu \mathrm{m} / \mathrm{min}$. As can be seen from these results the rate of size reduction of the Agave fibers decreases in each pulping stage due to the gradual lignin removal from the fibers. It is also, possible that some fibers and parenchymatic cells that cover the bundle of fibers were released during this process. Thereby, the results described above provided valuable information to a better understanding of the delignification stages occurring throughout the pulping of agave fibers. 


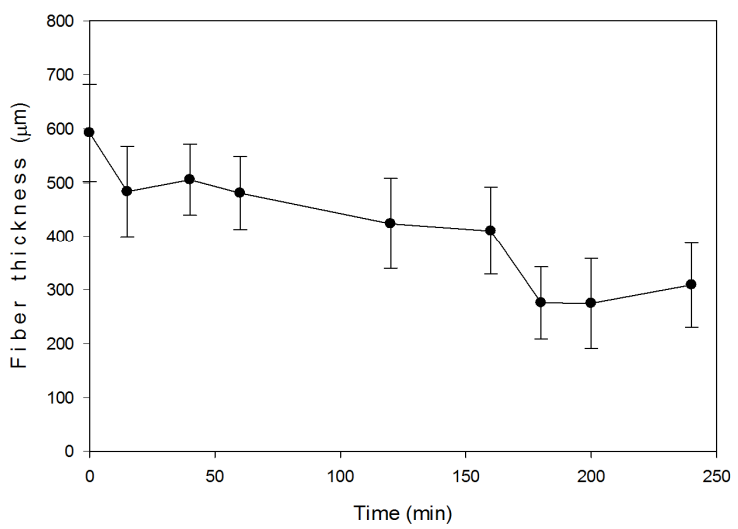

Fig. 3. Reduction of agave fibers thickness as function of cooking time

\subsection{Linear Prediction Models for Delignification of Agave Fibers by Image Analysis}

An attractive issue in the pulping process could be the prediction of delignification degree by applying a non-destructive method and by using a small quantity of sample. For this purpose CLSM images can be useful to evaluate the delignification of fibers by means of prediction models based on imaging techniques. Figure 4 shows an image gallery which illustrates the procedure performed to analyze CLSM images; here RGB images of lignin and cellulose were turned to gray level (GL) format in order to obtain GL and entropy (E) parameters. Then, GL images were converted to binary images to determinate the area fraction values. Thereby, RGB and grey level images were used for monitoring the delignification process. In Figure 4 is also possible to

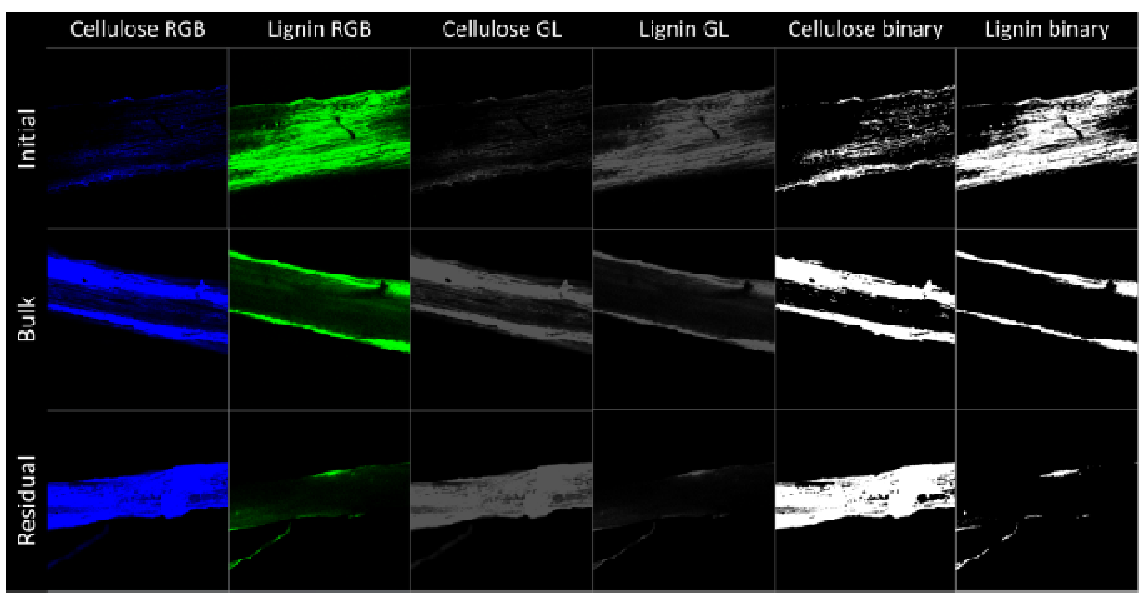

Fig. 4. Image gallery for image analysis of CLSM micrographs 

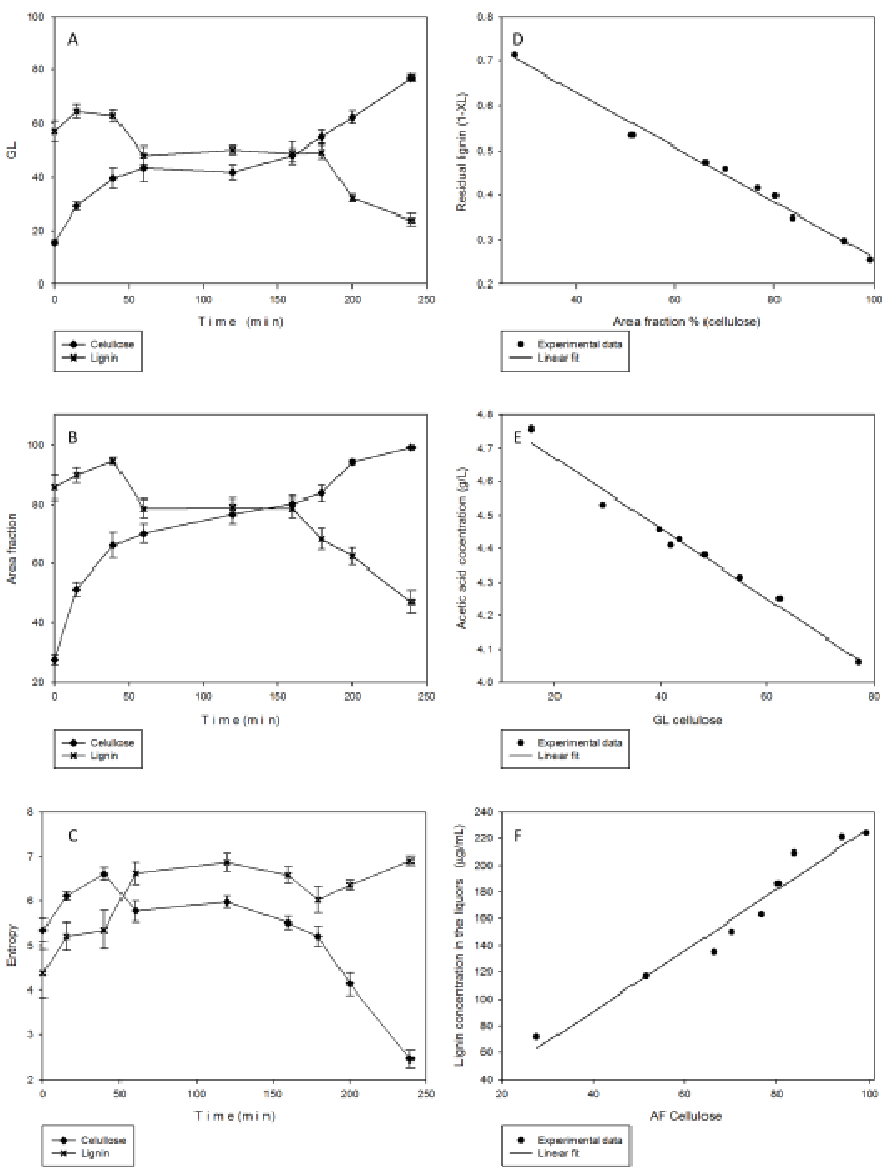

Fig. 5. Changes of imaging parameters during delignification process and relationships with chemical parameters. A, B and C kinetics of imaging parameters and D, E and F selected relationships between chemical and imaging parameters. AAC: acid acetic concentration, LC: lignin concentration in the liquors; RL: residual lignin; GL: grey level and AF: area fraction.

appreciate that as function of the reaction time, the cellulose fluorescence increased while the lignin autofluorescence decreased and a larger fluorescence can be associated with a higher concentration of lignin or cellulose.

Figure 5 shows the kinetics of GL, AF (area fraction) and E for cellulose and lignin components. GL parameter can be related with the intensity of fluorescence, while AF with the abundance or density of the fluorescence and consequently with lignin or cellulose contents. In GL and AF kinetics an increment on the cellulose fluorescence intensity with respect to the reaction time were observed (Figures $5 \mathrm{~A}$ and $5 \mathrm{~B}$ ) indicating a high content of cellulose in the fibers, while the lignin fluorescence, as measured by GL and AF parameters decreased as the reaction time proceeds; thus indicating a major delignification degree. Furthermore, image entropy, which is associated with the homogeneity 
of the gray level of the images, $[11,14]$ can be linked to fluorescence saturation of the images, as observed in Figure 5C, where the entropy values for cellulose decreased with the reaction time, while entropy values for lignin increased, indicating the elimination of lignin and the purification of cellulose on the fibers. This agreed with previous works, where has been assumed that the fluorescence of lignified materials is mainly attributable to the lignin content and its intensity is directly proportional with its concentration [3]. Also imaging parameters for cellulose and lignin were consistent with the three stages observed in chemical reaction kinetics and they provided quantitative information for monitoring of delignification process of agave fibers.

For identifying the best relationships between chemical and imaging parameters a Pearson correlation matrix was carried out (data not shown). Statistical analysis provides that all variables were significant, except the correlation between LC and entropy values for cellulose images. In overall, GL and AF for cellulose showed higher correlations ( $\mathrm{R}>0.956$ ) with chemical parameters (AAC, LC and RL). Thereby, cellulose images provided the better correlations between imaging and chemical parameters.

A selection of the best relationships is illustrated in Figure 5 D, E and F. For cellulose is clear that when the concentration of acetic acid decreased, GL and AF parameters increased (Figure 5D and E). Also for cellulose, the relationship between residual lignin and AF (Figure 5F), shows that as RL in the agave fibers decreased, AF increased. These relationships indicate that it is possible to directly associate the acid acetic uptake and the delignification degree of agave fibers with the fluorescence intensity (GL parameter) and image saturation (AF). According to the values obtained for $\mathrm{R}^{2}$ (Table 2), the better models for prediction of chemical parameters were RLAF, AAC-GL and LC-AF. The linear models proposed in the present work could be useful for prediction delignification level of agave fibers from CLSM images and by using a small quantity of sample.

Table 1. Selected linear prediction models for delignification degree from imaging parameters. AAC: acid acetic concentration, LC: lignin concentration in the liquors; RL: residual lignin; GL: grey level and AF: area fraction.

\begin{tabular}{ccc}
\hline Correlation & Best fit equation & $\mathbf{R}^{2}$ \\
\hline RL-AF & $R L=-0.0062 A F+0.8779$ & 0.989 \\
AAC-GL & $A A C=-0.0106 G L+4.8836$ & 0.981 \\
LC-AF & $L C=2.2806 A F-0.2808$ & 0.955 \\
AAC-AF & $A A C=-0.0084 A F+5.0044$ & 0.929 \\
LC-GL & $L C=2.7344 G L+38.9312$ & 0.913 \\
RL-GL & $R L=-0.0108 G L+0.8438$ & 0.911 \\
\hline
\end{tabular}




\section{Conclusions}

The delignification kinetics showed the existence of three periods: initial, bulk and residual stage, where a diminishing on the reaction rate was observed. Regarding microscopy studies, CLSM and IA allowed a quantitative evaluation of the delignification process, showing GL and AF parameters of cellulose a very good correspondence with chemical kinetic studies, resulting in very good linear prediction models. The data obtained shows that CLSM and IA can be used as a useful non-destructive methodology for the evaluation of the pulping of non-wood materials by using only a small quantity of fibers.

Acknowledgements. Hilda M. Hernandez wishes to thanks CONACyT and PIFI-IPN for the scholarship provided. This research was financial through the projects 20131864, 20130333 at the Instituto Politécnico Nacional (IPN-Mexico) and from CONACyT 161793, 133102. Cátedra Coca-Cola Jóvenes Investigadores 2011.

\section{References}

1. Narváez-Zapata, J.A., Sánchez-Teyer, L.F.: Agaves as a raw material: recent technologies and applications. Recent Patents on Biotechnology 3, 185-191 (2009)

2. Idarraga, G., Ramos, J., Zuniga, V., Sahin, T., Young, R.A.: Pulp and paper from blue agave waste from tequila production. Journal of Agricultural and Food Chemistry 47, 4450-4455 (1999)

3. Ma, J.F., Yang, G.H., Mao, J.Z., Xu, F.: Characterization of anatomy, ultrastructure and lignin microdistribution in Forsythia suspense. Industrial Crops and Products 33, 358-363 (2011)

4. Kestur, G.S., Flores-Sahagun, T.H.S., Dos Santos, L.P., Dos Santos, J., Mazzaro, I., Mikowski, A.: Characterization of blue agave bagasse fibers of Mexico. Composites: Part A 45, 153-161 (2013)

5. Thygesen, L.G., Hoffmeyer, P.: Image analysis for the quantification of dislocations in hemp fibres. Industrial Crops and Products 21, 173-184 (2005)

6. Quevedo, R., Mendoza, F., Aguilera, J.M., Chanona, J., Gutiérrez-López, G.: Determination of senescent spotting in banana Musa cavendish using fractal texture Fourier image. Journal of Food Engineering 84, 509-515 (2008)

7. Perea-Flores, M.J., Garibay-Febles, V., Chanona-Pérez, J.J., Calderón-Domínguez, G., Méndez-Méndez, J.V., Palacios-González, E., Gutiérrez-López, G.F.: Mathematical modelling of castor oil sedes (Ricinus communis) drying kinetics in fluidized bed at high temperatures. Industrial Crops and Products 38, 64-71 (2012)

8. Soudham, P.R., Rodríguez, D., Rocha, G.J.M., Taherzadeh, M.J., Martín, C.: Acetosolv delignification of marabou (Dichrostachys cinerea) wood with and without acid prehydrolysis. Forestry Studies in China 13(1), 64-70 (2011)

9. Farrera-Rebollo, R.R., Salgado-Cruz, M.P., Chanona-Pérez, J.J., Gutiérrez-López, G.F., Alamilla-Beltrán, L., Calderón-Domínguez, G.: Evaluation of image analysis tools for characterization of sweet bread crumb structure. Food Bioprocess Technology 5, 474-484 (2012) 
10. Haralick, R.M., Shanmugam, K., Dinstein, I.: Textural features for image classification. IEEE Transactions on Systems, Man and Cybernetics SMC 3(6), 610-621 (1973)

11. Arzate-Vázquez, I., Chanona-Pérez, J.J., Calderón-Domínguez, G., Terres-Rojas, E., Garibay-Febles, V., Martínez-Rivas, A., Gutiérrez-López, G.F.: Microstructural characterization of chitosan and alginate films by microscopy techniques and texture image analysis. Carbohydrate Polymers 87, 289-299 (2012)

12. Santos, A., Rodríguez, F., Gilarranz, M.A., Moreno, D., Garcia-Ochoa, F.: Kinetic modeling of kraft delignification of Eucalyptus globulus. Industrial \& Engineering Chemistry Research 36(10), 4114-4125 (1997)

13. Santos, R.B., Jameel, H., Chang, H., Hart, P.W.: Kinetics of Hardwood Carbohydrate Degradation during Kraft Pulp Cooking. Industrial \& Engineering Chemistry Research 51, 12192-12198(2012)

14. Mendoza, F., Dejmek, P., Aguilera, J.M.: Colour and image texture analysis in classification of commercial potato chips. Food Research International 40, 1146-1154 (2007) 